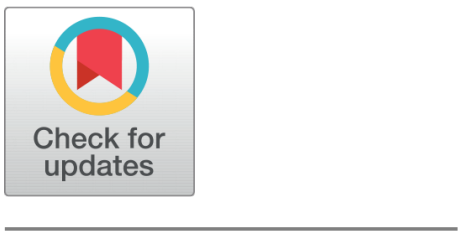

open ACCESS

Received: 04.06.2020

Accepted: 26.04 .2021

Published: 15.05 .2021

Citation: Singla N, Kalra S (2021) A two-dimensional Multiserver Queuing system with repeated attempts and impatience. Indian Journal of Science and Technology 14(17): 1379-1391. https://doi.org/ 10.17485/IJST/v14i17.820

* Corresponding author. soniakalra276@gmail.com

Funding: None

Competing Interests: None

Copyright: () 2021 Singla \& Kalra. This is an open access article distributed under the terms of the Creative Commons Attribution License, which permits unrestricted use, distribution, and reproduction in any medium, provided the original author and source are credited.

Published By Indian Society for Education and Environment (iSee)

ISSN

Print: 0974-6846

Electronic: 0974-5645

\section{A two-dimensional Multiserver Queuing system with repeated attempts and impatience}

\author{
Neelam Singla ${ }^{1}$, Sonia Kalra ${ }^{2 *}$ \\ 1 Assistant Professor, Department of Statistics, Punjabi University, Patiala, 147001, India \\ 2 Research Scholar, Department of Statistics, Punjabi University, Patiala, 147001, India
}

\section{Abstract}

Objective: This study discusses a two-state multiserver retrial queueing system, where the customer may leave the system due to impatience. In this paper, we deal with the time dependent probabilities when all, some or none servers are busy. Method: For this model, we solved difference differential equations recursively and obtained the time dependent probabilities when all, some or none servers are busy. Findings: Time dependent probabilities of exact number of arrivals and exact number of departures at when all, some or none servers are busy are obtained. In this paper, some kind of verification and converting two state model into single state model are discussed. Some special cases of interest are also discussed. Novelty: In communication networks, multiple servers are used to reduce traffic congestion and improve system performance. The operation mode of a call center with repeated attempts provides an initial motivation for our study.

Keywords: Impatience; Multiserver; Probability; Queueing; Retrial

\section{Introduction}

Retrial queueing models serve as the quantitative technique in evaluating the operating performance of call centers. Retrial queues (queueing systems with repeated attempts, or queues with returning customers) are characterized by the following feature: a request on finding all servers busy upon arrival leaves the service area, but repeats his demand after some (random) time. For example, if the control tower can not accept request for landing from an aircraft, then later has to circle in the sky. The aircraft repeats its request until the runway is available to it for landing. Retrial queues are widely and successfully used as mathematical models for several computer systems and telecommunication networks. For a comprehensive review of the main results and literature relating to retrials, the reader is referred to the papers Artalejo ${ }^{(1)}$, Falin ${ }^{(2)}$, Kulkarni \& Liang ${ }^{(3)}$, Yang \& Templeton ${ }^{(4)}$.

In communication networks, multiple servers are used to reduce traffic congestion and improve system performance. The operation mode of a call center with repeated attempts provides an initial motivation for our study. In a queueing model of a call center, the customers are callers, the servers are telephone agents (operators), and 
queues are populated by callers that wait for service. The operation of the call center can be described as follows: when a customer call arrives, it will be served immediately if a server is available. If all servers are busy with other calls, the customer makes repeated attempts until the connection is made. The call center may choose to announce an expected waiting time to the customer at this point. Some customers are patient enough to wait for a server to become available. Other customers will hang up immediately upon hearing the waiting time announcement or abandon after waiting for some time. However, multiserver queues with impatience, have attracted much attention in queueing literature because of explosive demands to efficiently design and manage call centers. Therefore, in this paper, we present a multiserver queueing model with customer's retrials and impatience.

The problem of queues with impatient customers was first analyzed by Palm ${ }^{(5)}$. Motivated by the impact of impatience and repeated calls in the conventional systems, Cohen ${ }^{(6)}$ studied a multiserver queue with retrial pool and no waiting positions. Baccelli et al. ${ }^{(7)}$ studied the waiting time distribution in $\mathrm{M} / \mathrm{M} / \mathrm{c}$ queue with general impatience bound on queueing times by constructing a simple Markov process. Perel and Yechiali ${ }^{(8)}$ considered a two-phase service model where the customers become impatient. Choudhary and Medhi ${ }^{(9)}$ studied a multiserver queueing model with reneging and balking where the reneging rate is not constant

The classical transient results for the $\mathrm{M} / \mathrm{M} / 1$ queue provide a little insight into the behavior of a queueing system through fixed operation time $t$, but provide virtually no information on how the system has operated until time $t$. To answer the questions regarding the system operation during time period $t$, we developed a two - dimensional $\mathrm{M} / \mathrm{M} / \mathrm{c}$ retrial queueing model with impatient customers in which the state of the system is given by $(i, j)$, where $i$ describes the exact number of arrivals in the system and $j$ describes the exact number of departures from the system until time $t$. The transient analysis of this model helps us to understand the behavior of a system. Pegden and Rosenshine ${ }^{(10)}$ analyzed the M/M/1 two-state queueing model. Indra and Ruchi ${ }^{(11)}$ obtained two-dimensional state time dependent probabilities along with some interesting particular cases for a single server markovian queueing system where the service mechanism was non-exhaustive. Garg and Kumar ${ }^{(12)}$ obtained explicit time dependent probabilities of exact number of arrivals and departures from the orbit of a single server retrial queue with impatient customers.

There are many more researchers who did the similar kind of study but the major difference to my study is we studied the twodimensional state retrial queueing model instead of total number of customers in the system. We also obtained time dependent probabilities of the exact number of arrivals and exact number of departures at when all, some or none servers are busy from the system instead of the orbit.

The rest of the paper is organized as follows: The description of the underlying queueing model and the derivation of the difference-differential equations are done in Section 2. The time dependent solution for the model is obtained in Section 3. In Section 4, some important performance measures and special cases are obtained. Concluding remarks are presented in Section 5.

\section{Model Description}

The service facility consists of a group of ${ }^{\prime} c^{\prime}$ fully available channels where primary calls arrive. Arrival of primary calls follow a Poisson stream with rate $\lambda$. If an arriving caller finds some channel free, it immediately occupies the channel, takes the service and then leaves the system. If all the servers are busy at the time of primary calling, the caller leaves the system without service with probability $\left(1-a_{1}\right)$ and joins the orbit with probability $a_{1}>0$ to retry for service from there. Repeating callers retries for getting service from the orbit and following a Poisson process with parameter $\theta$. If an incoming repeated call finds the server free, it joins the server and leaves the system after being served. Otherwise, if the server is busy at the time of repeated calling, there are two possibilities either the caller leaves the system/orbit without service due to impatience with probability $\left(1-a_{2}\right)$ or retries again for service with probability $a_{2}>0$. Service times are exponentially distributed with service rate $\mu$. Interarrival times, service times, inter-impatient times involved in the above description are assumed to be statistically independent.

Laplace transformation $\bar{f}$ (s) of $f(t)$ is given by

$$
\bar{f}(\mathrm{~s})=\int_{0}^{\infty} e^{-s t} f(t) \mathrm{dt}, \quad \operatorname{Re}(\mathrm{s})>0
$$

The Laplace inverse of

$$
\frac{Q(p)}{P(p)} \text { is } \sum_{k=1}^{n} \sum_{l=1}^{m_{k}} \frac{t^{m_{k}-l} e^{a_{k} t}}{\left(m_{k}-l\right) !(l-1) !} X \frac{d^{l-1}}{d p^{l-1}}\left(\frac{Q(p)}{P(p)}\right)\left(p-a_{k}\right)^{m_{k}}, \forall p=a_{k}, \quad a_{i} \neq a_{k} \text { for } i \neq k .
$$


Where,

$$
\begin{aligned}
& P(p)=\left(p-a_{1}\right)^{m_{1}}\left(p-a_{2}\right)^{m_{2}} \ldots \ldots \ldots\left(p-a_{n}\right)^{m_{n}} \\
& Q(p) \text { is a polynomial of degree }<m_{1}+m_{2}+m_{3}+\ldots \ldots \ldots \ldots m_{n}-1
\end{aligned}
$$

The Laplace inverse of $\bar{N}_{n_{1, n_{2}, n_{3}}}^{a, b, c}(\mathrm{~s})=\frac{1}{(s+a)^{n_{1}}(s+b)^{n_{2}}(s+c)^{n_{3}}}$ is

$P(p)=\left(p-a_{1}\right)^{m_{1}}\left(p-a_{2}\right)^{m_{2}} \ldots \ldots \ldots\left(p-a_{n}\right)^{m_{n}}$

$Q(p)$ is a polynomial of degree $<m_{1}+m_{2}+m_{3}+\ldots \ldots \ldots \ldots m_{n}-1$.

The Laplace inverse of $\bar{N}_{n_{1, n_{2}, n_{3}}}^{a, b, c}(\mathrm{~s})=\frac{1}{(s+a)^{n_{1}}(s+b)^{n_{2}}(s+c)^{n_{3}}}$ is

$$
\begin{aligned}
& N_{n_{1, n_{2}, n_{3}}}^{a, b, c}(t)=\sum_{l=1}^{n_{3}} \sum_{m=1}^{l} \frac{e^{-a t} t^{n_{3}-l}(-1)^{m+1}\left(\frac{l-1}{m-1}\right)\left(\prod_{g_{1}=0}^{l-m-1}\left(n_{1}+g_{1}\right)\right)\left(\prod_{g_{2}=0}^{m-2}\left(n_{2}+g_{2}\right)\right)}{\left(n_{3}-l\right) !(m-1) !(b-a)^{n_{2}+m-1}(c-a)^{n_{1}+l-m}} \\
& +\sum_{l=1}^{n_{2}} \sum_{m=1}^{l} \frac{e^{-b t} t^{n_{2}-l}(-1)^{m+1}\left(\frac{l-1}{m-1}\right)\left(\prod_{g_{1}=0}^{l-m-1}\left(n_{1}+g_{1}\right)\right)\left(\prod_{g_{2}=0}^{m-2}\left(n_{3}+g_{2}\right)\right)}{\left(n_{2}-l\right) !(m-1) !(a-b)^{n_{3}+m-1}(c-b)^{n_{1}+l-m}} \\
& +\sum_{l=1}^{n_{1}} \sum_{m=1}^{l} \frac{e^{-c t} t^{n_{1}-l}(-1)^{m+1}\left(\frac{l-1}{m-1}\right)\left(\prod_{g_{1}=0}^{l-m-1}\left(n_{2}+g_{1}\right)\right)\left(\prod_{g_{2}=0}^{m-2}\left(n_{3}+g_{2}\right)\right)}{\left(n_{1}-l\right) !(m-1) !(a-c)^{n_{3}+m-1}(b-c)^{n_{2}+l-m}}
\end{aligned}
$$

If $L^{-1}\{f(s)\}=F(t)$ and $L^{-1}\{g(s)\}=G(t)$, then

$L^{-1}\{f(s) g(s)\}=\int_{0}^{t} F(u) G(t-u) \mathrm{du}=F * G, F * G$ is called the convolution of $F$ and $G$.

\subsection{The Two-Dimensional State Model}

An $\mathrm{M} / \mathrm{M} / \mathrm{c}$ queue is a stochastic process whose state space is the set $(0,1,2,3 \ldots \ldots\}$ where the values correspond to the number of arrivals enter in the system being served and depart from the system. This model includes features that are quite general, and as a result, a rather extensive class of well-known and often applied queueing systems can be viewed as simply special cases of the fundamental birth-and-death model.

The model assumes a queueing system with $c(c=1,2,3 \ldots)$ parallel identical servers and infinite system capacity, operating in the following fashion:

1. The arrival of primary calls follows a Poisson distribution with parameter $\lambda$ and move the process from state $i$ to $i+1$.

2. The repeated calls to each server follow a Poisson distribution with parameter $\theta$.

3. Service times for each server are exponentially distributed with parameter $\mu$ and move the process from state $j$ to $j-1$.

4. The stochastic processes involved viz. arrivals of units, departures of units and retrials are statistically independent.

\section{Definitions}

$P_{i, j, 0}(t)=$ Probability that there are exactly $i$ arrivals in the system and $j$ departures from the system by time $t$ when server is idle.

$P_{i, j, m}(t)=$ Probability that there are exactly $i$ arrivals in the system and $j$ departures from the system by time $t$ when $m$ servers are busy. $1 \leq m \leq c-1$.

$P_{i, j, c}(t)=$ Probability that there are exactly $i$ arrivals in the system and $j$ departures from the system by time $t$ when all the $c$ servers are busy.

$P_{i, j}(t)=$ Probability that there are exactly $i$ arrivals in the system and $j$ departures from the system by time $t$.

$$
P_{i, j}(t)=P_{i, j, 0}(t)+\sum_{m=1}^{c-1} P_{i, j, m}(t)+P_{i, j, c}(t), \forall i, j \quad i \geq j
$$

also

Initially

$$
P_{i, j, c}(t)=0 \& P_{i, j, m}(t)=0 \text { for } i \leq j, 1 \leq m \leq c-1 ; P_{i, j, 0}(t)=0, i<j
$$

$$
P_{0,0,0}(0)=1 ; P_{i, j, 0}(0)=0, P_{i, j, c}(0)=0 \& P_{i, j, m}(t)=0, \forall i, j \neq 0 \& 1 \leq m \leq c-1
$$




\subsection{The difference - differential equations governing the system are}

$$
\begin{aligned}
& \frac{d}{d t} P_{i, j, 0}(t)=-(\lambda+(i-j) \theta) P_{i, j, 0}(t)+\mu P_{i, j-1,1}(t) \quad i \geq j \geq 0 \\
& \frac{d}{d t} P_{i, j, m}(t)=-(\lambda+m \mu+(i-j-m) \theta) P_{i, j, m}(t)+\lambda P_{i-1, j, m-1}(t)+ \\
& (i-j-(m-1)) \theta P_{i, j, m-1}(t)+(m+1) \mu P_{i, j-1, m+1}(t) \\
& i>j \geq 0,1 \leq m<c \\
& \frac{d}{d t} P_{i, j, c}(t)=-\left(\lambda a_{1}+c \mu+(i-j-c) \theta\left(1-a_{2}\right)\right) P_{i, j, c}(t)+\lambda P_{i-1, j, c-1}(t)+\lambda a_{1}(1- \\
& \left.\delta_{i-c, j}\right) P_{i-1, j, c}(t)+(i-j-(c-1)) \theta P_{i, j, c-1}(t)+(i-j-(c-1)) \theta\left(1-a_{2}\right) P_{i, j-1, c}(t) \\
& i>1, i>j \geq 0 \\
& \text { where } \delta_{i-c, j}=\left\{\begin{array}{l}
1, \text { when } i-c=j \\
0, \text { otherwise }
\end{array}\right.
\end{aligned}
$$

Using the Laplace transformation $\bar{f}(\mathrm{~s})$ of $f(t)$ given by

$$
\bar{f}(s)=\int_{0}^{\infty} e^{-s t} f(t) \mathrm{dt}, \quad \operatorname{Re}(\mathrm{s})>0
$$

in the equations (2.1) - (2.3) along with the initial conditions, we have

$$
\left.\begin{array}{c}
(s+\lambda+(i-j) \theta) \bar{P}_{i, j, 0}(s)=\mu \bar{P}_{i, j-1,1}(s) \quad i \geq j \geq 0 \\
(s+\lambda+m \mu+(i-j-m) \theta) \bar{P}_{i, j, m}(s)=\lambda \bar{P}_{i-1, j, m-1}(s)+(i-j(m-1)) \theta \bar{P}_{i, j, m-1}(s)+ \\
(m+1) \mu \bar{P}_{i, j,-1, m+1}(s) \\
i>j \geq 0,1 \leq m<c
\end{array}\right)
$$

\section{Solution of the Problem}

Solving equations (2.4) to (2.6) recursively, we have

$$
\begin{gathered}
\bar{P} 0,0,0(\mathrm{~s})=\frac{1}{s+\lambda} \\
\bar{P}_{i, i, 0}(s)=\frac{\mu}{(s+\lambda)} \bar{P}_{i, i-1,1}(s) \quad \text { for } i \geq 1 \\
\bar{P}_{m, 0, m}(s)=\frac{\lambda}{s+\lambda+m \mu} \bar{P}_{m-1,0, m-1}(s) \quad \text { for } 1 \leq m \leq c-1 \\
\bar{P}_{i, i-m, m}(s)=\frac{\lambda \quad \bar{P}_{i-1, i-m, m-1}(s)+\frac{(m+1) \mu}{s+\lambda+m \mu} \bar{P}_{i, i-m-1, m+1}(s)}{s+\lambda+m \mu} \\
\bar{P}_{c, 1, c-1}(s)=\frac{\lambda=1 \text { to } c-2, i=m+1 \text { to } c-1}{(s+\lambda+(c-1) \mu)} \bar{P}_{c-1,1, c-2}(s)+\frac{c \mu}{(s+\lambda+(c-1) \mu)} \bar{P}_{c, 0, c}(s)
\end{gathered}
$$




$$
\begin{gathered}
\bar{P}_{i, 1, c-1}(s)=\frac{c \mu}{(s+\lambda+(c-1) \mu+(i-j-(c-1)) \theta} \prod_{p=0}^{i-c} \frac{\lambda^{i-(c-1)} a_{1}^{i-c}}{\left(s+\lambda a_{1}+c \mu+p \theta\left(1-a_{2}\right)\right)^{m}} \bar{P}_{c-1,0, c-1}(s) \\
\text { for } i>c
\end{gathered}
$$

for $i \geq(c-1)+j, j>1$

$$
\begin{aligned}
& \text { where } \eta_{\mathrm{k}}^{\prime}(\mathrm{s})=\left\{\begin{array}{cl}
1 & \text { for } k=1 \\
\left(1+\frac{(k-1) \theta a_{1}}{\left(s+\lambda a_{1}+c \mu+(k-2) \theta\left(1-a_{2}\right)\right)}\right) & \text { for } k=2 \text { to } i-j-(c-2) \\
\frac{(k-1) \theta}{\left(s+\lambda a_{1}+c \mu+(k-2) \theta\left(1-a_{2}\right)\right)} & \text { for } k=i-j-(c-3)
\end{array}\right. \\
& \bar{P}_{i, j, m}(s)=\frac{\lambda}{(s+\lambda+m \mu+(i-j-m) \theta} \bar{P}_{i-1, j, m-1}(s)+\frac{(i-j-(m-1)) \theta}{(s+\lambda+m \mu+(i-j-m) \theta} \bar{P}_{i, j, m-1} \\
& \frac{(m+1) \mu}{(s+\lambda+m \mu+(i-j-m) \theta}\left\{\frac{\lambda}{(s+\lambda+(m+1) \mu+(i-j-m) \theta} \bar{P}_{i-1, j-1, m}(s)+\right. \\
& \left.\begin{array}{r}
\frac{(i-j-(m-1)) \theta}{(s+\lambda+(m+1) \mu+(i-j-m) \theta} \bar{P}_{i, j-1, m}(s)+\frac{(m+2) \mu}{(s+\lambda+(m+1) \mu+(i-j-m) \theta} \bar{P}_{i, j-2, m+2}(s) \\
\text { for } 1 \leq m \leq \mathrm{c}-2, i \geq j+m, j>c-m
\end{array}\right\} \\
& \bar{P}_{i, j, 0}(s)=\frac{(m+1) \mu}{s+\lambda+(i-j) \theta}\left[\begin{array}{c}
\frac{\lambda}{s+\lambda+\mu+(i-j) \theta} \bar{P}_{i-1, j-1,0}(s)+\frac{(i-j+1) \theta}{s+\lambda+\mu+(i-j) \theta} \bar{P}_{i, j-1,0}(s)+ \\
\frac{\lambda}{s+\lambda+\mu+(i-j) \theta} \\
\frac{(i-j+1) \theta}{\vdots+\lambda+2 \mu+(i-j) \theta} \bar{P}_{i, j-1, m+1}(s)+ \\
\frac{(m+3) \mu}{s+\lambda+2 \mu+(i-j) \theta} \bar{P}_{i-1, j-2, m+1}(s)+ \\
\frac{(m+\lambda+2 \mu+(i-j) \theta}{s+\lambda} \bar{P}_{i, j-3, m+3}(s)
\end{array}\right\}
\end{aligned}
$$

for $i>j \geq \mathrm{c}$ 
Taking the Inverse Laplace transform of equations (3.1) to (3.11), we have

$$
\begin{aligned}
& P_{0,0,0}(t)=e^{-\lambda t} \\
& P_{i, i, 0}(t)=\mu e^{-\lambda t} * P_{i, i-1,1}(t) \quad \text { for } i \geq 1 \\
& P_{m, 0, m}(t)=\lambda e^{-(\lambda+m \mu) t} * P_{m-1,0, m-1}(t) \quad \text { for } 1 \leq m \leq \mathrm{c}-1 \\
& \begin{array}{c}
P_{i, i-m, m}(t)=\lambda e^{-(\lambda+m \mu) t} * P_{i-1, i-m, m-1}(t)+(m+1) \mu e^{-(\lambda+m \mu) t} * P_{i, i-m-1, m+1}(t) \\
\text { for } m=1 \text { to } c-2, i=m+1 \text { to } c-1
\end{array} \\
& P_{c, 1, c-1}(t)=\lambda e^{-(\lambda+(c-1) \mu) t} * P_{c-1,1, c-2}(t)+c \mu e^{-(\lambda+(c-1) \mu) t} * P_{c, 0, c}(t) \\
& P_{i, 1, c-1}(t)=\mathrm{c} \mu \lambda^{i-(c-1)} a_{1}^{i-c} e^{-(\lambda+(c-1) \mu+(i-j-(c-1)) \theta) t} \\
& \left\{\prod_{p=0}^{i-c} \frac{1}{\left(\frac{c \mu}{a_{1}}+\frac{p \theta\left(1-a_{2}\right)}{a_{1}}\right)}-\frac{e^{-\left(\frac{c \mu}{a_{1}}+\frac{p \theta\left(1-a_{2}\right)}{a_{1}}\right) t}}{\left(\frac{c \mu}{a_{1}}+\frac{p \theta\left(1-a_{2}\right)}{a_{1}}\right)}\right\} * P_{c-1,0, c-1}(t) \text { fori }>c \\
& P_{i, 0, c}(t)=\lambda^{i-(c-1)} a_{1}{ }^{i-c}\left\{\prod_{p=0}^{i-c} e^{-\left(\lambda a_{1}+c \mu+p \theta\left(1-a_{2}\right)\right)}\right\} * P_{c-1,0, c-1}(t) \\
& \text { for } i \geq \mathrm{c} \\
& P_{i, j, c}(t)=\lambda^{i-j-(c-1)} a_{1}{ }^{i-j-c}\left\{\prod_{p=0}^{i-j-c} e^{-\left(\lambda a_{1}+c \mu+p \theta\left(1-a_{2}\right)\right) t}\right\} * P_{j+c-1, j, c-1}(t)+ \\
& \sum_{k=2}^{i-j-(c-1)} \lambda^{i-j-(c-2)-k} a_{1}^{i-j-(c-2)-(k+1)}\left\{\prod_{p=k-1}^{i-j-c} e^{-\left(\lambda a_{1}+c \mu+p \theta\left(1-a_{2}\right)\right) t}\right\} \\
& * P_{j+k+c-2, j, c-1}(t)+\sum_{k=2}^{i-j-(c-1)} \lambda^{i-j-(c-2)-k} a_{1}^{i-j-(c-2)-(k+1)}+ \\
& (k-1) \theta a_{1} e^{-\left(\lambda a_{1}+c \mu+(k-2) \theta\left(1-a_{2}\right)\right) t}\left\{\prod_{p=k-1}^{i-j-c} \frac{1}{\left(\frac{c \mu}{a_{1}}+\frac{p \theta\left(1-a_{2}\right)}{a_{1}}\right)}-\frac{e^{-\left(\frac{c \mu}{a_{1}}+\frac{p \theta\left(1-a_{2}\right)}{a_{1}}\right) t}}{\left(\frac{c \mu}{a_{1}}+\frac{p \theta\left(1-a_{2}\right)}{a_{1}}\right)}\right\} \\
& * P_{j+k+c-2, j, c-1}(t)+\frac{(i-j-c+1) \theta}{a_{1}} e^{-\left(\lambda a_{1}+c \mu+(i-j-c) \theta\left(1-a_{2}\right)\right) t} P_{i, j, c-1}(t)+ \\
& \sum_{k=1}^{i-j-(c-1)}\left(\lambda a_{1}\right)^{i-j-(c-1)-k} k \theta\left(1-a_{2}\right)\left\{\prod_{p=k-1}^{i-j-c} e^{-\left(\lambda a_{1}+c \mu+p \theta\left(1-a_{2}\right)\right) t}\right\} \\
& * P_{j+k+c-1, j-1, c}(t) \quad \text { for } i \geq j+c, j \geq 1 \\
& P_{i, j, c-1}(t)=\left(\lambda e^{-(\lambda+(c-1) \mu+(i-j-(c-1)) \theta) t}\right) * P_{i-1, j, c-2}(t)+(i-j-(c-2)) \theta \\
& \left(e^{-(\lambda+(c-1) \mu+(i-j-(c-1)) \theta) t}\right) * P_{i, j, c-2}(t)+(c \mu) \lambda^{i-j-(c-2)} \\
& -(\lambda+(c-1) \mu+(i-j-(c-1)) \theta) t\left\{\Pi_{p=0}^{i-j-(c-1)} \frac{1}{\left(\frac{c \mu}{a_{1}}+\frac{p \theta\left(1-a_{2}\right)}{a_{1}}\right)}-\frac{e^{-\left(\frac{c \mu}{a_{1}}+\frac{p \theta\left(1-a_{2}\right)}{a_{1}}\right) t}}{\left(\frac{c \mu}{a_{1}}+\frac{p \theta\left(1-a_{2}\right)}{a_{1}}\right)}\right\} \\
& * P_{j+2, j-1, c-1}(t)+(c \mu) e^{-(\lambda+(c-1) \mu+(i-j-(c-1)) \theta) t} \sum_{k=2}^{i-j-c+2} \lambda^{i-j-(c-3)-k} \\
& a_{1}^{i-j-(c-3)-(k+1)}\left\{\prod_{p=k-1}^{i-j-(c-1)} \frac{1}{\left(\frac{c \mu}{a_{1}}+\frac{p \theta\left(1-a_{2}\right)}{a_{1}}\right)}-\frac{e^{-\left(\frac{c \mu}{a_{1}}+\frac{p \theta\left(1-a_{2}\right)}{a_{1}}\right) t}}{\left(\frac{c \mu}{a_{1}}+\frac{p \theta\left(1-a_{2}\right)}{a_{1}}\right)}\right\} *
\end{aligned}
$$


$P_{j+k+1, j-1, c-1}(t)+$

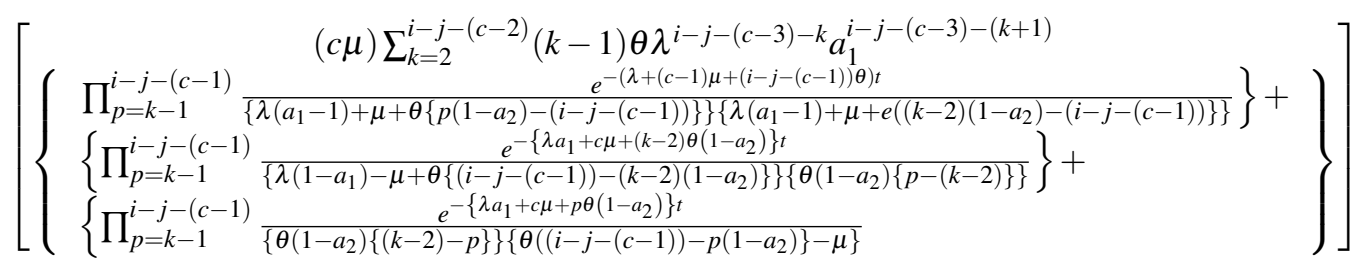

$$
\begin{aligned}
& * P_{j+k+1, j-1, c-1}(t)+\frac{c \mu(i-j-c+2) e^{-\left(\lambda a_{1}+(c-1) \mu+(i-j-(c-1)) \theta\right) t}}{a_{1}} \\
& \left\{\frac{1}{\left(\frac{c \mu}{a_{1}}+\frac{(i-j-c+1) \theta\left(1-a_{2}\right)}{a_{1}}\right)}-\frac{e^{-\left(\frac{c \mu}{a_{1}}+\frac{(i-j-c+1) \theta\left(1-a_{2}\right)}{a_{1}}\right) t}}{\left(\frac{c \mu}{a_{1}}+\frac{(i-j-c+1) \theta\left(1-a_{2}\right)}{a_{1}}\right)}\right\} * P_{i-c+4, j-1, c-1}(t)+c \mu \\
& e^{-(\lambda+(c-1) \mu+(i-j-(c-1)) \theta) t} \sum_{k=2}^{i-j-c+2}\left(\lambda a_{1}\right)^{i-j-(c-2)-k} k \theta(1- \\
& \left.a_{2}\right)\left\{\prod_{p=k-1}^{i-j-(c-1)} \frac{1}{\left(\frac{c \mu}{a_{1}}+\frac{\left.p \theta_{1}-a_{2}\right)}{a_{1}}\right)}-\frac{e^{-\left(\frac{c \mu}{a_{1}}+\frac{p \theta\left(1-a_{2}\right)}{a_{1}}\right) t}}{\left(\frac{c \mu}{a_{1}}+\frac{p \theta\left(1-a_{2}\right)}{a_{1}}\right)}\right\} * P_{j+k+c-2, j-2, c}(t)
\end{aligned}
$$

for $i \geq(c-1)+j, j \geq 1$

$$
\begin{gathered}
P_{i, j, m}(t)=\lambda e^{-\{\lambda+m \mu+(i-j-m) \theta\} t} * P_{i-1, j, m-1}(t)+(i-j-(m-1) \theta) \\
e^{-\{\lambda+m \mu+(i-j-m) \theta\} t} * P_{i, j, m-1}(t)+\lambda((m+1) \mu) e^{-\{\lambda+m \mu+(i-j-m) \theta\} t} \\
\left\{\frac{1}{\{(m+1) \mu+(i-j-m) \theta\}}-\frac{e^{-\{(m+1) \mu+(i-j-m) \theta\} t}}{((m+1) \mu+(i-j-m) \theta\}}\right\} * P_{i-1, j-1, m}(t)+(m+1) \mu \\
(i-j-(m-1)) \theta e^{-\{\lambda+m \mu+(i-j-m) \theta\} t} \\
\left\{\frac{1}{\{(m+1) \mu+(i-j-m) \theta\}}-\frac{e^{-\{(m+1) \mu+(i-j-m) \theta\} t}}{\{(m+1) \mu+(i-j-m) \theta\}}\right\} * P_{i, j-1, m}(t)+ \\
(m+1)(m+2) \mu^{2} e^{-\{\lambda+m \mu+(i-j-m) \theta\} t}\left\{\frac{1}{\{(m+1) \mu+(i-j-m) \theta\}}\right. \\
\left.-\frac{e^{-\{(m+1) \mu+(i-j-m) \theta\} t}}{\{(m+1) \mu+(i-j-m) \theta\}}\right\} * P_{i, j-2, m+2}(t) \\
\text { for } 1 \leq m \leq c-2, i \geq j+m, j \geq(c-m)
\end{gathered}
$$




$$
\begin{gathered}
P_{i, j, 0}(t)=\left[\lambda(m+1) \mu e^{-(\lambda+(i-j) \theta\} t}\left\{\frac{1}{\mu+(i-j) \theta\}}-\frac{e^{-\{\mu+(i-j) \theta\} t}}{\{\mu+(i-j) \theta\}}\right\}\right] * P_{i-1, j-1,0}(t)+ \\
\quad\left[((i-j+1) \theta)(m+1) \mu e^{-(\lambda+(i-j) \theta\} t}\left\{\frac{1}{\mu+(i-j) \theta\}}-\frac{e^{-\{\mu+(i-j) \theta\} t}}{\{\mu+(i-j) \theta\}}\right\}\right] * P_{i, j-1,0}(t) \\
+\left[(m+1)(m+2) \mu^{2} \lambda\left\{\frac{e^{-\{\lambda+(i-j) \theta\} t}}{2 \mu^{2}}+\frac{e^{-\{\lambda+\mu+(i-j) \theta\} t}}{\mu^{2}}-\frac{e^{-\{\lambda+(i-j) \theta+2 \mu\} t}}{2 \mu^{2}}\right\}\right] \\
* P_{i-1, j-2, m+1}(t)+\left[( m + 1 ) ( m + 2 ) \mu ^ { 2 } ( ( i - j + 1 ) \theta ) \left\{\frac{e^{-\{\lambda+(i-j) \theta\} t}}{2 \mu^{2}}+\right.\right. \\
\left.\quad \frac{e^{-\{\lambda+\mu+(i-j) \theta\} t}}{\mu^{2}}-\frac{e^{-\{\lambda+(i-j) \theta+2 \mu\} t}}{2 \mu^{2}}\right\} * P_{i, j-2, m+1}(t)+ \\
{\left[(m+1)(m+2)(m+3) \mu^{3}\left\{\frac{e^{-\{\lambda+(i-j) \theta\} t}}{2 \mu^{2}}+\frac{e^{-\{\lambda+\mu+(i-j) \theta\} t}}{\mu^{2}}-\frac{e^{-\{\lambda+(i-j) \theta+2 \mu\} t}}{2 \mu^{2}}\right\}\right]} \\
* P_{i, j-3, m+3}(t) \quad \\
\text { for } i>j \geq \mathrm{c}
\end{gathered}
$$

\section{Some Important Performance Measures}

\subsection{The Laplace transform of the probability $P_{i .}(t)$ that exactly $i$ units arrive by time $t$ is :}

$$
\bar{P}_{i .}(s)=\sum_{j=0}^{i} \bar{P}_{i, j}(s)=\frac{\lambda^{i}}{(s+\lambda)^{i+1}} ; \quad i>0
$$

And its Inverse Laplace transform is

$$
P_{i .}(t)=\frac{e^{-\lambda t}(\lambda t)^{i}}{i !}
$$

The very (basic) assumption on primary arrivals is that it forms a Poisson process and above analysis of abstract solution also verifies the same.

4.2 The probability that exactly $j$ customers have been served by time $t, P_{. j}(t)$ in terms of $P_{i, j}(t)$ is given by:

$$
P_{. j}(t)=\sum_{i=j}^{\infty} P_{i, j}(t)
$$

4.3 From the abstract solution of our model, we verified that the sum of all possible probabilities is one i.e. taking summation over $i$ and $j$ on equations (3.1) $-(3.11)$ and adding, we get

$$
\sum_{i=0}^{\infty} \sum_{j=0}^{i}\left\{\bar{P}_{i, j, 0}(s)+\sum_{m=1}^{c-1} \bar{P}_{i, j, m}(s)+\bar{P}_{i, j, c}(s)\right\}=\frac{1}{s} .
$$

After taking the inverse Laplace transformation, we get

$$
\sum_{i=0}^{\infty} \sum_{j=0}^{i}\left\{P_{i, j, 0}(t)+\sum_{m=1}^{c-1} P_{i, j, m}(t)+P_{i, j, c}(t)\right\}=1 .
$$


which is a verification of our results.

\subsection{Define $Q_{n, m}(t)$ as the probability that there are $n$ customers in the orbit at time $t$ and $m \quad(m=1,2, \ldots c)$ servers are busy.}

When $m$ servers are busy, it is defined by probability $Q_{n, m}(t)$

$$
Q_{n, m}(t)=\sum_{j=0}^{\infty} P_{j+n+m, j, m}(t) \quad(m=1,2, \ldots c)
$$

The number of customers i.e. ' $n$ ' in the orbit is obtained by using the relation:

$n=$ (number of arrivals - number of departures $-m)$

Using above relation and letting $\mu=1$ from the equations (2.1) to (2.3) the set of equations in statistical equilibrium are:

$$
\begin{gathered}
(\lambda+m+n \theta) Q_{n, m}=\lambda Q_{n, m-1}+(n+1) \theta Q_{n+1, m-1}+(m+1) Q_{n, m+1} \\
0 \leq m \leq c-1, n \geq 0 \\
\left(\lambda a_{1}+n \theta\left(1-a_{2}\right)+c\right) Q_{n, c}=\lambda Q_{n, c-1}+(n+1) \theta Q_{n+1, c-1} \\
+\lambda a_{1} Q_{n-1, c}(1-\delta n, 0)+(n+1) \theta\left(1-a_{2}\right) Q_{n+1, c} \\
(\text { case } m=c), n \geq 0 \\
\text { where } \delta_{n, 0}=\left\{\begin{array}{l}
1, \text { when } n=0 \\
0, \text { when } n \geq 1
\end{array}\right.
\end{gathered}
$$

Now, for generating functions

$$
Q_{m}(z)=\sum_{n=0}^{\infty} z^{n} Q_{n, m} \quad 0 \leq m \leq c
$$

Letting $z=1$, then $Q_{m}(1)=\sum_{n=0}^{\infty} Q_{n, m}=Q_{m}$ (say) $0 \leq m \leq c$

these equations become

$$
\begin{gathered}
(\lambda+m) Q_{m}(z)+\theta z Q_{m}^{\prime}(z)=\lambda Q_{m-1}(z)+\theta Q_{m-1}^{\prime}(z)+(m+1) Q_{m+1}(z) \\
0 \leq m \leq c-1 \\
\lambda a_{1}(1-z) Q_{c}(z)+c Q_{c}(z)=\lambda Q_{c-1}(z)+\theta Q_{c-1}^{\prime}(z)+\theta\left(1-a_{2}\right)(1-z) Q_{c}^{\prime}(z) \\
(\text { case } m=c)
\end{gathered}
$$

Then above equations coincide with the equations of (4.17) and (4.18) of Falin \& Templeton ${ }^{(13)}$.

\subsection{Special Cases:}

(a) When we consider $a_{1}=1$ and $a_{2}=1$ in equations (4.3) and (4.4), we get

$$
\begin{aligned}
& (\lambda+m+n \theta) Q_{n, m}=\lambda Q_{n, m-1}+(n+1) \theta Q_{n+1, m-1}+(m+1) Q_{n, m+1} \\
& 0 \leq m \leq c-1, n \geq 0 \\
& (\lambda+c) Q_{n, c}=\lambda Q_{n, c-1}+(n+1) \theta Q_{n+1, c-1}+\lambda Q_{n-1, c}\left(1-\delta_{n, 0}\right) \\
& \text { (case } m=c \text { ), } n \geq 0 \text {. } \\
& \text { where } \delta_{n, 0}=\left\{\begin{array}{l}
1, \text { when } n=0 \\
0, \text { when } n \geq 1
\end{array}\right.
\end{aligned}
$$

Above equations coincide with that of (2.17) and (2.18) of Falin \& Templeton ${ }^{(13)}$. 
(b) Letting $a_{1}=\beta, a_{2}=1$ and $c=1$ in equations (3 12) to (3 22), we get the following equations

$$
\begin{aligned}
& P_{0,0,0}(t)=e^{-\lambda t} \\
& P_{i, 1,0}(\mathrm{t})=\mu e^{-(\lambda+(i-1) \theta) t} * P_{i, 0,1}(\mathrm{t}) \quad \text { for } i \geq 1 \\
& P_{i, i, 0}(\mathrm{t})=\left[(\lambda \mu) e^{-\lambda t}\left\{\frac{1}{\left(\frac{\mu}{\beta}\right)}-\frac{e^{-\left(\frac{\mu}{\beta}\right) t}}{\left(\frac{\mu}{\beta}\right)}\right\} * P_{i-1, i-1,0}(\mathrm{t})+(\mu \theta) e^{-\lambda t}\left\{\frac{1}{\left(\frac{\mu}{\beta}\right)}-\frac{e^{-\left(\frac{\mu}{\beta}\right) t}}{\left(\frac{\mu}{\beta}\right)}\right\} * P_{i, i-1,0}(\mathrm{t})\right] \\
& P_{1,0,1}(\mathrm{t})=\lambda e^{-\lambda t}\left\{\frac{1}{\left(\frac{\mu}{\beta}\right)}-\frac{e^{-\left(\frac{\mu}{\beta}\right) t}}{\left(\frac{\mu}{\beta}\right)}\right\} \\
& P_{i, 0,1}(\mathrm{t})=(\lambda \beta)^{i-1} e^{-(\lambda \beta+\mu) t} \frac{t^{i-2}}{(i-2) !} * P_{1,0,1}(t) \quad \text { for } i>1 \\
& P_{i, i-1,1}(\mathrm{t})=\left(\lambda e^{-(\lambda \beta+\mu) t} * P_{i-1, i-1,0}(\mathrm{t})+\theta e^{-(\lambda \beta+\mu) t} * P_{i, i-1,0}(\mathrm{t})\right) \\
& P_{i, j, 1}(t)={ }^{i-j-1}(\beta)^{i-j-2} e^{-(\beta+) t} \frac{t^{i-j-2}}{(i-j-2) !} * P_{j+1, j, 0}(t)+ \\
& \sum_{k=2}^{i-j-1}\left[i-j-k(\beta)^{i-j-k-1} e^{-(\beta+) t} \frac{t^{i-j-k-1}}{(i-j-k-1) !} * P_{j+k, j, 0}(t)\right]+ \\
& \sum_{k=2}^{i-j-1}\left[i-j-k(\beta)^{i-j-k-1}(k \theta \beta) e^{-(\beta+) t} \frac{t^{i-j-k}}{(i-j-k) !} * P_{j+k, j, 0}(t)\right]+ \\
& (i-j) \theta e^{-(\beta+) t} * P_{i, j, 0}(t)+(\beta)^{i-j-1} e^{-(\beta+) t} \frac{t^{i-j-2}}{(i-j-2) !} * P_{j+1, j, 1}(t) \\
& P_{i, j, 0}(t)=\mu \lambda^{i-j}(\beta)^{i-j-1} e^{-(\lambda+(i-j) \theta) t}\left\{\begin{array}{l}
\text { for } i \geq j+2, j \geq 1 \\
\frac{1}{\left(\frac{\mu}{\beta}\right)^{i-j}}-e^{-\left(\frac{\mu}{\beta}\right) t} \sum_{r=0}^{i-j-1} \frac{t^{r}}{r !} \frac{1}{\left(\frac{\mu}{\beta}\right)^{i-j-r}}
\end{array}\right\} * \\
& P_{j, j-1,0}(t)+\mu e^{-(\lambda+(i-j) \theta) t}\left[\sum_{k=2}^{i-j}(\lambda)^{i-j-k+1}(\beta)^{i-j-k}\left\{\frac{1}{\left(\frac{\mu}{\beta}\right)^{i-j-k+1}}-e^{-\left(\frac{\mu}{\beta}\right) t} \sum_{r=0}^{i-j-k} \frac{t^{r}}{r !} \frac{1}{\left(\frac{\mu}{\beta}\right)^{i-j-k+1-r}}\right\} * P_{j+k-1, j-1,0}(t)\right]+
\end{aligned}
$$

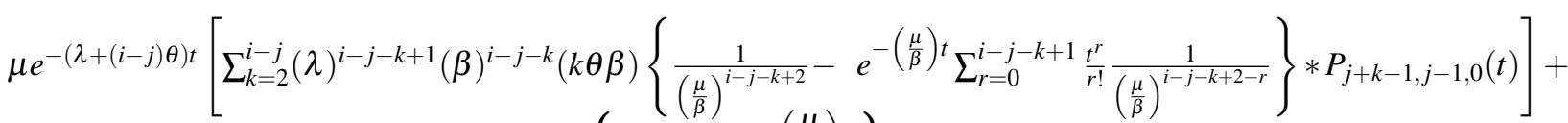

$$
\begin{aligned}
& \mu(i-j+1) \theta e^{-(\lambda+(i-j) \theta) t}\left\{\frac{1}{\left(\frac{\mu}{\beta}\right)}-\frac{e^{-\left(\frac{\mu}{\beta}\right) t}}{\left(\frac{\mu}{\beta}\right)}\right\} P_{i, j-1,0}(t)+ \\
& \mu(\lambda \beta)^{i-j} e^{-(\lambda+(i-j) \theta) t}\left\{\frac{1}{\left(\frac{\mu}{\beta}\right)^{i-j}}-e^{-\left(\frac{\mu}{\beta}\right) t i-j-1} \sum_{r=0}^{i-\frac{t^{r}}{r !}} \frac{1}{\left(\frac{\mu}{\beta}\right)^{i-j-r}}\right\} * P_{j, j-1,1}(t) \\
& \text { for } i>j>1
\end{aligned}
$$

Above equation coincide with those of Singla and Kalra ${ }^{(14)}$. 
(c) On putting $a_{1}=1, a_{2}=\alpha$ and $c=1$ in equations (3.12) to (3.22), we get the following equations and these results coincide with that of Singla and Kalra ${ }^{(15)}$.

$$
\left.\begin{array}{c}
P_{0,0,0}(t)=e^{-\lambda t} \\
P_{i, 1,0}(t)=\mu e^{-(\lambda+(i-1) \theta) t} * P_{i, 0,1}(t) \quad \text { for } i \geq 1 \\
P_{i, i, 0}=\left[\begin{array}{c}
\left.(\lambda \mu) e^{-\lambda t}\left\{\frac{1}{\mu}-\frac{e^{-\mu t}}{\mu}\right\} * P_{i-1, i-1,0}(t)+(\mu \theta) e^{-\lambda t}\left\{\frac{1}{\mu}-\frac{e^{-\mu t}}{\mu}\right\} * P_{i, i-1,0}(t)\right] \\
+(\mu \theta)(1-\alpha) e^{-\lambda t}\left\{\frac{1}{\mu}-\frac{e^{-\mu t}}{\mu}\right\} * P_{i, i-2,1}(t) \\
\text { for } i>1
\end{array}\right] \\
P_{1,0,1}(t)=\lambda e^{-\lambda t}\left\{\frac{1}{\mu}-\frac{e^{-\mu t}}{\mu}\right\} \\
P_{i, i-1,1}(\mathrm{t})=\left(\lambda e^{-(\lambda+\mu) t} * P_{i-1, i-1,0}+\theta e^{-(\lambda+\mu) t} * P_{i, i-1,0}+\theta(1-\alpha) e^{-(\lambda+\mu) t} *\right. \\
\left.\left.P_{i, i-2,1}\right) \quad \begin{array}{c}
\text { for } i>1 \\
\text { for } i \geq j+2, j \geq 1
\end{array}\right]+(\lambda)^{i-1}\left\{\prod_{m=1}^{i-1} e^{-(\lambda+\mu+m \theta(1-\alpha)) t} * P_{1,0,1}(t)\right. \\
P_{i, j, 1}(t)=\lambda^{i-j-1}\left\{\prod_{m=1}^{i-j-1} e^{-(\lambda+\mu+m \theta(1-\alpha)) t}\right\} \frac{t^{m-1}}{(m-1) !} * P_{j+1, j, 0}(t)+ \\
\sum_{k=2}^{i-j-1}\left[\lambda^{i-j-k}\left\{\prod_{m=k}^{i-j-1} e^{-(\lambda+\mu+m \theta(1-\alpha)) t}\right\} \frac{t^{m-k}}{(m-k) !} * P_{j+k, j, 0}(t)\right]+ \\
\sum_{k=2}^{i-j-1}\left[\lambda^{i-j-k}(k \theta)\left\{\prod_{m=k-1}^{i-j-1} e^{-(\lambda+\mu+m \theta(1-\alpha)) t}\right\} \frac{t^{m-k}}{(m-k) !} * P_{j+k, j, 0}(t)\right]+ \\
(i-j) \theta e^{-(\lambda+\mu+(i-j-1) \theta(1-\alpha)) t} * P_{i, j, 0}(t)+\sum_{k=1}^{i-j-1}\left[(\lambda)^{i-j-k-1}(k+1) \theta\right. \\
\left.(1-\alpha)\left\{\prod_{m=k}^{i-j-1} e^{-(\lambda+\mu+m \theta(1-\alpha)) t}\right\} \frac{t^{m-k}}{(m-k) !} * P_{j+k+1, j-1,1}(t)\right]+ \\
\left.(\lambda)^{i-j-1}\left\{\prod_{p=1}^{i-j-1} e^{-(\lambda+\mu+p \theta(1-\alpha)) t}\right\} \frac{t^{p-1}}{(p-1) !} * P_{j+1, j, 1}(t)\right]
\end{array}\right]
$$




$$
\begin{gathered}
P_{i, j, 0}(t)=\mu \lambda^{i-j} e^{-(\lambda+(i-j) \theta) t}\left\{\prod_{m=1}^{i-j} \frac{1}{(\mu+m \theta(1-\alpha))^{m}}-e^{-(\mu+m \theta(1-\alpha)) t} \sum_{r=0}^{m-1} \frac{t^{r}}{r !} \frac{1}{(\mu+m \theta(1-\alpha))^{m-r}}\right\} * \\
P_{j, j-1,0}(t)+\lambda \mu e^{-(\lambda+(i-j) \theta) t}\left[\sum _ { k = 2 } ^ { i - j } ( \lambda ) ^ { i - j - k } \left\{\prod_{m=k}^{i-j} \frac{1}{(\mu+m \theta(1-\alpha))^{m-k+1}}-\right.\right. \\
\left.\left.e^{-(\mu+m \theta(1-\alpha)) t} \sum_{r=0}^{m-k} \frac{t^{r}}{r !} \frac{1}{(\mu+m \theta(1-\alpha))^{m-k+1-r}}\right\} * P_{j+k-1, j-1,0}(t)\right]+ \\
\lambda \mu e^{-(\lambda+(i-j) \theta) t}\left[\sum _ { k = 2 } ^ { i - j } ( \lambda ) ^ { i - j - k } ( k \theta ) \left\{\prod_{m=k-1}^{i-j} \frac{1}{(\mu+m \theta(1-\alpha))^{m-k+2}}-\right.\right. \\
e^{-(\mu+m \theta(1-\alpha)) t} \sum_{r=0}^{m-k+1} \frac{t^{r}}{r !} \frac{1}{\left.\left.(\mu+m \theta(1-\alpha))^{m-k+2-r}\right\} * P_{j+k-1, j-1,0}(t)\right]+\mu(i-j+} \\
1) \theta e^{-(\lambda+(i-j) \theta) t}\left\{\frac{1}{\mu+(i-j) \theta(1-\alpha)}-\frac{e^{-(\mu+(i-j) \theta(1-\alpha)) t}}{\mu+(i-j) \theta(1-\alpha)}\right\} * P_{i, j-1,0}(t)+ \\
\mu e^{-(\lambda+(i-j) \theta) t}\left[\sum _ { k = 1 } ^ { i - j } ( \lambda ) ^ { i - j - k } ( k + 1 ) \theta ( 1 - \alpha ) \left\{\prod_{m=k}^{i-j} \frac{1}{(\mu+m \theta(1-\alpha))^{m-k+1}}-\right.\right. \\
\left.\left.e^{-(\mu+m \theta(1-\alpha)) t} \sum_{r=0}^{m-k} \frac{t^{r}}{r !} \frac{1}{(\mu+m \theta(1-\alpha))^{m-k+1-r}}\right\} * P_{j+k, j-2,1}(t)\right]+ \\
\mu(\lambda)^{i-j} e^{-(\lambda+(i-j) \theta) t}\left\{\prod_{p=1}^{i-j} \frac{1}{(\mu+p \theta(1-\alpha))^{p}}-e^{-(\mu+p \theta(1-\alpha)) t} \sum_{r=0}^{p-1} \frac{t^{r}}{r !} \frac{1}{(\mu+p \theta(1-\alpha))^{p-r}}\right\} * P_{j, j-1,1}(t) \\
f o r i>j>1
\end{gathered}
$$

\section{Conclusion}

Customer impatience represents the loss in revenues and customer goodwill to the service provider. Multiple servers are used to reduce traffic congestion and also used in highly efficient bandwidth intensive applications. In this study, we considered a Makovian multiserver model, where the simultaneous effect of customer impatience and retffigrials is evaluated. Previous research helps us to deal with arrivals and departures from the orbit but in our research, we discuss about the arrivals and departures from the system instead of the orbit which makes a major impact on latest research. We have used the recursive method to find the time dependent probabilities when all, some or none servers are busy. In queueing theory generally, we deal with a total number of customers in the system but in this research, we deal with the two-dimensional state which means the exact number of arrivals in the system and exact number of departures from the system. After finding the probabilities, we verified our results with the help of these probabilities. Along with this some special cases also computed which matches with the previous research.

\section{References}

1) Artalejo JR. Accessible bibliography on retrial queues. Mathematical and Computer Modelling. 1999;30(3-4):1-6. Available from: https://dx.doi.org/10. 1016/s0895-7177(99)00128-4.

2) Falin G. A survey of retrial queues. Queueing Systems. 1990;7(2):127-167. Available from: https://dx.doi.org/10.1007/bf01158472.

3) Kulkarni VG, Liang H. Retrial queues Revisited. In: Dshalalow JH, editor. Frontiers in Queueing. CRC Press. 1998;p. 19-34. Available from: https://dl.acm.org/doi/10.5555/279251.279253.

4) Yang T, Templeton JGC. A survey on retrial queues. Queueing Systems. 1987;2(3):201-233. Available from: https://dx.doi.org/10.1007/bf01158899.

5) Palm C. Methods of judging the annoyance caused by congestion. Telecommunications. 1953;4:4-5. Available from: http://ie.technion.ac.il/serveng/ Lectures/PalmAnnoyance.pdf.

6) Cohen JW. Basic problems of telephone traffic theory and the influence of repeated calls. Philips Telecommunication Review. 1957;18:49-100. Available from: https://ci.nii.ac.jp/naid/10000050792/. 
7) Baccelli F, Boyer P, Hebuterne G. Single-server queues with impatient customers. Advances in Applied Probability. 1984;16(4):887-905. Available from: https://doi.org/10.2307/1427345.

8) Perel N, Yechiali U. Queues with slow servers and impatient customers. European Journal of Operational Research. 2010;201(1):247-258. Available from: https://dx.doi.org/10.1016/j.ejor.2009.02.024.

9) Choudhury A, Medhi P. Balking and reneging in multiserver Markovian queuing system. International Journal of Mathematics in Operational Research. 2011;3(4):377-394. Available from: https://dx.doi.org/10.1504/ijmor.2011.040874.

10) Pegden CD, Rosenshine M. Some New Results for theM/M/1 Queue. Management Science. 1982;28(7):821-828. Available from: https://dx.doi.org/10. $1287 / \mathrm{mnsc}$.28.7.821.

11) Indra, Ruchi. Transient analysis of two- dimensional state Markovian queueing model with multiple working vacations and non-exhaustive service. International Journal of Computer Applications. 2010;11(7):1-7. Available from: https://www.ijcaonline.org/archives/volume11/number7/1598-2147.

12) Garg PC, Kumar S. A Single Server Retrial Queue With Impatient Customers. Mathematical Journal of Interdisciplinary Sciences. 2012;1(1):67-82. Available from: https://dx.doi.org/10.15415/mjis.2012.11006.

13) Falin GI, Templeton JGC. Retrial Queues. 1st ed. and others, editor;Chapman and Hall/CRC. 1997. Available from: https://www.routledge.com/RetrialQueues/Falin-Templeton/p/book/9780412785504.

14) Singla N, Kalra S. A Two - State Retrial Queueing Model with Balking Customers. International Journal of Interdisciplinary Research and Innovations. 2018;6:528-539. Available from: https://researchpublish.com/journal-details/IJIRI.

15) Singla N, Kalra S. A Two - State Retrial Queueing Model with Reneging Customers. International Journal of Management. 2018;8:2650-2663. Available from: http://ijamtes.org/VOL-8-ISSUE-10-2018-1/. 